Advances in Dynamical Systems and Applications.

ISSN 0973-5321, Volume 16, Number 1, (2021) pp. 59-66

(C) Research India Publications

https://dx.doi.org/10.37622/ADSA/16.1.2021.59-66

\title{
Model to Study Pressure Behavior of Horizontal Well Subjected by Both Bottom Water and Gas Cap at Late Time
}

\author{
John Obarhire Oloro" ${ }^{* 1}$ and Ufuoma Kazeem Okpeki ${ }^{2}$ \\ ${ }^{1}$ Chemical and Petroleum Engineering, Faculty of Engineering, Oleh Campus, \\ Delta State University, Delta State, Nigeria \\ ${ }^{2}$ Electric/Electronic Engineering, Faculty of Engineering, Oleh Campus, \\ Delta State University, Delta State, Nigeria
}

\begin{abstract}
A model to study the behavior of pressure in a horizontal well subjected by both bottom water and Gas cap and bottom water drives at a late period has been developed using source function and numerical technique was used for the computation from the results. The following are pressure decreases with time to when $\mathrm{tD}=1000$ and pressure derivative also decrease to when $\mathrm{tD}=100$. The pressure became stable when $\mathrm{tD} \geqslant 100$ as shown in Figures 2,3 and 4. This behavior is an indication that reservoir fluid has encountered a boundary. From Figure 3 and 4, it was observed that the decline rate increases with time to a point when $\mathrm{tD} \geqslant 100$. At this point, the Gas cap which acts as a drive is not active. Hence, there will be no increase in production at this stage.
\end{abstract}

Keywords: pressure, horizontal well, model, gas cap, reservoir.

\section{INTRODUCTION}

The horizontal well Method is well well-known for enhancing the well productivity of low-permeability reservoirs, especially for reservoirs with a gas cap or bottom water [1-3]. Drilling horizontal wells are important to maximizing oil production compared to vertical wells as well as to lessen near-wellbore disorder in high and low permeability reservoir. The financial achievement with the horizontal wells not only large with reserves but also the production is of a short period compared to vertical wells [4]. In this work, a model would be developed. The objective is to study the Pressure behavior of a horizontal well that is subjected to both bottom water and gas cap at a late time and 
determine the effect of some well parameters.

\subsection{Description of the model}

Figure 1 represents the 3D of the reservoir model and axial description of the reservoir of length xe in the x-direction, length ye in the y-direction, and length $\mathrm{z}$ in z-direction subjected by Top Gas cap and bottom water. It has horizontal well positions yw, xw, and $\mathrm{zW}$ in $\mathrm{y}, \mathrm{x}$, and $\mathrm{z}$ directions respectively.

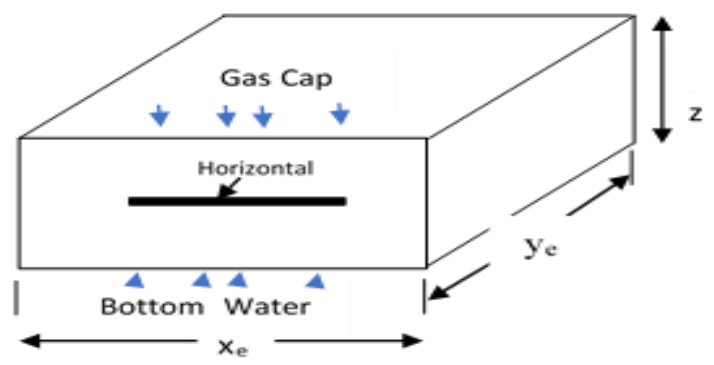

Fig. 1 Reservoir sealed at $x$ and $y$ axis's and subjected by Top Gas cap and Bottom water [2]

\section{MATERIALS AND METHODS}

To study the behaviors of pressure in a horizontal well subjected by Both Bottom Water and Gas Cap at a Late Time was done by following the methodology process [5 - 8]. Fig. 2 represent the 4 steps

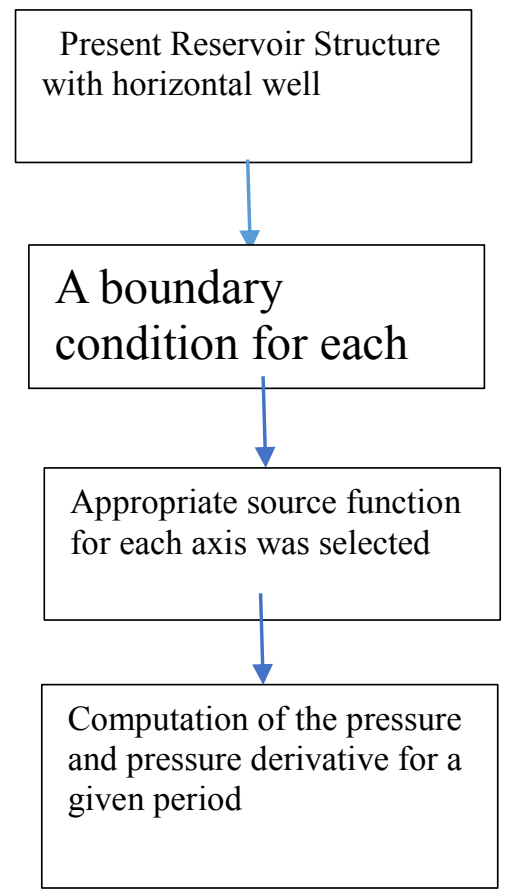

Fig.2 Behavior study of pressure in a horizontal well subjected by Both Bottom Water and Gas Cap at Late Time methodology 
From Figure 1, at the x-position, well encounter an infinite slab source from an infinite slab reservoir in which the top is a constant-pressure boundary [7-8]. Hence, the appropriate

$$
\begin{aligned}
& S\left(x_{D}, t_{D}\right)=\frac{2}{x_{e D}}\left\{1+\frac{2 x_{e D}}{\pi} \sum_{n=1}^{\infty} \frac{1}{n} \exp \left[-\frac{n^{2} x^{2} t_{D}}{x_{e D}}\right] \sin \frac{n \pi x_{w D}}{x_{e D}} \cos \frac{n \pi x_{w D}}{x_{e D}} \cos \frac{n \pi x_{D}}{x_{e D}}\right\} \\
& S\left(y_{D}, t_{D}\right)=\frac{1}{y_{D}}\left\{1+2 \sum_{n=1}^{\infty} \frac{1}{n} \exp \left[-\frac{m^{2} \pi^{2} t_{D}}{y_{D}}\right] \cos \frac{m \pi y_{w D}}{y_{D}} \cos \frac{m \pi y_{D}}{y_{D}}\right\} \\
& S\left(z_{D}, t_{D}\right)=\frac{1}{h_{D}}\left\{1+2 \sum_{n=1}^{\infty} \frac{1}{n} \exp \left[-\frac{l^{2} \pi^{2} t_{D}}{h_{D}}\right] \sin \frac{l \pi z_{D}}{z_{D}} \sin \frac{l \pi z_{w D}}{h_{D}}\right\}
\end{aligned}
$$

Putting equations 12 and 3 in 4 we have Equation 5 as the pressure distribution for reservoir sealed at $\mathrm{x}$ and $\mathrm{y}$ axes and subjected by top Gas cap and bottom water. This model (Equation 5) Reservoir with underlain water and Top gas cap.

$$
\begin{aligned}
& P_{D}=2 \pi h_{D} \int_{0}^{t_{D}} S\left(x_{D}, t_{D}\right) S\left(y_{D}, t_{D}\right) S\left(z_{D}, \tau\right) \partial t_{D} \\
& P_{D}=2 \pi h_{D}\left[\frac{2}{x_{e D}} * \frac{1}{y_{D}}\left\{1+2 \sum_{n=1}^{\infty} \frac{1}{n} \exp \left[-\frac{m^{2} \pi^{2} t_{D}}{y_{D}}\right] \cos \frac{m \pi y_{w D}}{y_{D}} \cos \frac{m \pi y_{D}}{y_{D}}\right\} *\right. \\
& \frac{1}{h_{D}}\left\{1+2 \sum_{n=1}^{\infty} \frac{1}{n} \exp \left[-\frac{l^{2} \pi^{2} t_{D}}{h_{D}}\right] \sin \frac{l \pi z_{D}}{h_{D}} \sin \frac{l \pi z_{w D}}{h_{D}}\right\}
\end{aligned}
$$

Equation 5 is the dimensionless pressure for the reservoir system. The dimensionless pressure derivative is given as

$p_{D}^{\prime}=t_{D} \frac{\partial p_{D}}{\partial t_{D}}$

Using Equation 6, Equation 7. The dimensionless pressure derivative of Equation 5 is given as $\left.P_{D}^{\prime}=\frac{4 \pi}{x_{e D}} \frac{1}{y_{D}}\right\rfloor \quad\{1+$

$\left.\frac{2 x_{e D}}{\pi} \sum_{n=1}^{\infty} \frac{1}{n} \exp \left[-\frac{n^{2} x^{2} D_{D}}{x_{e D}}\right] \sin \frac{n \pi x_{w D}}{x_{e D}} \cos \frac{n \pi x_{w D}}{x_{e D}} \cos \frac{n \pi x_{D}}{x_{e D}}\right\} *\{1+$

$\left.2 \sum_{n=1}^{\infty} \frac{1}{n} \exp \left[-\frac{m^{2} \pi^{2} t_{D}}{y_{D}}\right] \cos \frac{m \pi y_{w D}}{y_{D}} \cos \frac{m \pi y_{D}}{y_{D}}\right\} * \frac{1}{h_{D}}\{1+$

$\left.2 \sum_{n=1}^{\infty} \frac{1}{n} \exp \left[-\frac{l^{2} \pi^{2} t_{D}}{h_{D}}\right] \sin \frac{l \pi z_{D}}{z_{D}} \sin \frac{l \pi z_{w D}}{h_{D}}\right\}$

\section{RESULTS AND DISCUSSIONS}

Equation 5 is solved using the Gauss-Legendre quadrature, a numerical method at the final flow period8. Equation 7 does not need any integration but the direct substitution of reservoir system parameters is assumed. The solutions are presented in Table 1. 
Table 1: Horizontal well $\left(X e_{D}, X D, X w D, y D, Y w D, Z w D, z D, h D\right)$ : $(6,0.25,1,0.74,0.74,0.3,0.55,30)$

\begin{tabular}{|l|l|l|}
\hline $\mathrm{Td}$ & PD & PD' \\
\hline 0.001 & 29.74 & $2.23 \mathrm{E}-01$ \\
\hline $1.00 \mathrm{E}-02$ & $2.85 \mathrm{E}+01$ & $2.10 \mathrm{E}-01$ \\
\hline 0.1 & $2.09 \mathrm{E}+01$ & $1.41 \mathrm{E}-01$ \\
\hline 1 & $1.45 \mathrm{E}+01$ & $1.05 \mathrm{E}-01$ \\
\hline 10 & $1.02 \mathrm{E}+01$ & $6.74 \mathrm{E}-02$ \\
\hline 100 & $5.92 \mathrm{E}+00$ & $4.25 \mathrm{E}-02$ \\
\hline 1000 & $5.66 \mathrm{E}+00$ & $4.25 \mathrm{E}-02$ \\
\hline 10000 & $5.66 \mathrm{E}+00$ & $4.25 \mathrm{E}-02$ \\
\hline
\end{tabular}

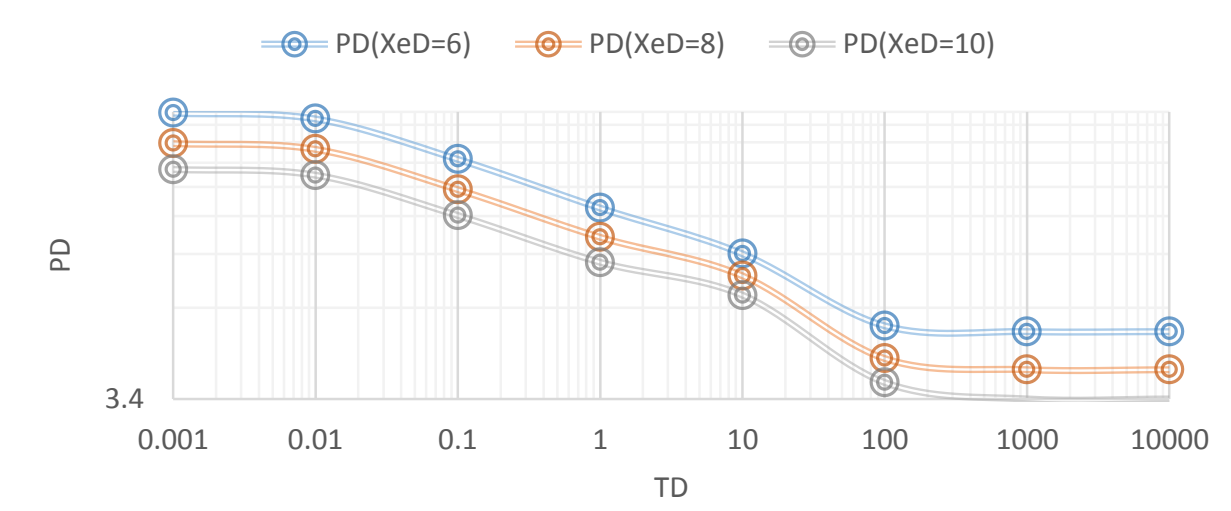

Fig. 3 Effect of $X$ on Dimensionless Pressure

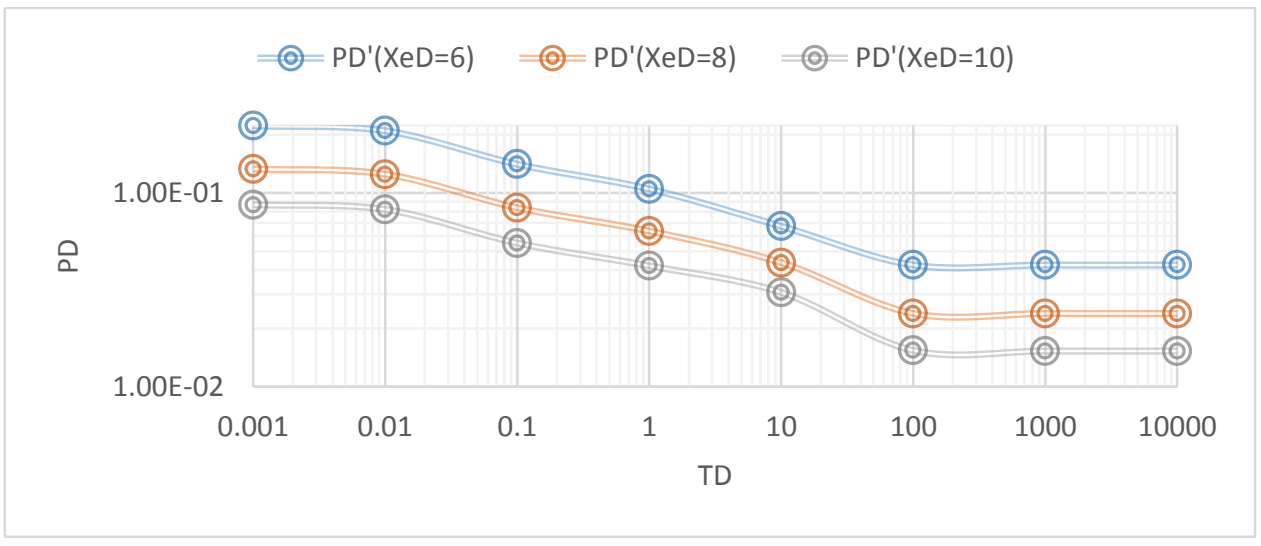

Fig. 4 Effect of $X_{e D}$ on Dimensionless Pressure Derivative 
Table 2: Effect of yeD on Dimensionless Pressure and Dimensionless Pressure Derivative

\begin{tabular}{|c|c|c|c|c|c|c|}
\hline$T d$ & $P D(h D=30)$ & $\begin{array}{c}\mathrm{PD}^{\prime} \\
(\mathrm{hD}=30)\end{array}$ & $\begin{array}{c}\mathrm{PD} \\
(\mathrm{hD}=20)\end{array}$ & $\begin{array}{c}\mathrm{PD}^{\prime} \\
(\mathrm{hD}=20)\end{array}$ & $\mathrm{PD}(\mathrm{XeD}=10)$ & $\mathrm{PD}^{\prime}(\mathrm{XeD}=10)$ \\
\hline 0.001 & 29.74 & $2.23 \mathrm{E}-01$ & 29.74 & $3.34 \mathrm{E}-01$ & 29.74 & $6.69 \mathrm{E}-01$ \\
\hline $1.00 \mathrm{E}-02$ & $2.85 \mathrm{E}+01$ & $2.10 \mathrm{E}-01$ & $2.85 \mathrm{E}+01$ & $3.15 \mathrm{E}-01$ & $2.85 \mathrm{E}+01$ & $6.31 \mathrm{E}-01$ \\
\hline 0.1 & $2.09 \mathrm{E}+01$ & $1.41 \mathrm{E}-01$ & $2.09 \mathrm{E}+01$ & $2.11 \mathrm{E}-01$ & $2.09 \mathrm{E}+01$ & $4.23 \mathrm{E}-01$ \\
\hline 1 & $1.45 \mathrm{E}+01$ & $1.05 \mathrm{E}-01$ & $1.45 \mathrm{E}+01$ & $1.58 \mathrm{E}-01$ & $1.45 \mathrm{E}+01$ & $3.16 \mathrm{E}-01$ \\
\hline 10 & $1.02 \mathrm{E}+01$ & $6.74 \mathrm{E}-02$ & $1.02 \mathrm{E}+01$ & $1.01 \mathrm{E}-01$ & $1.02 \mathrm{E}+01$ & $2.02 \mathrm{E}+00$ \\
\hline 100 & $5.92 \mathrm{E}+00$ & $4.25 \mathrm{E}-02$ & $5.92 \mathrm{E}+00$ & $6.38 \mathrm{E}-02$ & $5.92 \mathrm{E}+00$ & $1.28 \mathrm{E}-01$ \\
\hline 1000 & $5.66 \mathrm{E}+00$ & $4.25 \mathrm{E}-02$ & $5.66 \mathrm{E}+00$ & $6.38 \mathrm{E}-02$ & $5.66 \mathrm{E}+00$ & $1.28 \mathrm{E}-01$ \\
\hline 10000 & $5.66 \mathrm{E}+00$ & $4.25 \mathrm{E}-02$ & $5.66 \mathrm{E}+00$ & $6.38 \mathrm{E}-02$ & $5.66 \mathrm{E}+00$ & $1.28 \mathrm{E}-01$ \\
\hline
\end{tabular}

Table 2 shows the effect of $y_{\mathrm{eD}}$ on Dimensionless Pressure and Dimensionless Pressure Derivative. This was done by altering the value of $\mathrm{y}_{\mathrm{eD}}$ with time and the result shows that $\mathrm{y}_{\mathrm{eD}}$ does not vary Dimensionless Pressure and Dimensionless Pressure Derivative for horizontal well subjected to Gas cap at a late period. Table 3 shows the variation of $\mathrm{h}_{\mathrm{D}}$ on Dimensionless Pressure and Dimensionless Pressure Derivative. This was also This was done by varying the value of $h_{D}$ with time and the result shows that $h_{D}$ does not affect Dimensionless Pressure as shown in Figure 5, but it affects Dimensionless Pressure Derivative for horizontal well subjected to Gas cap at a late period as shown in Figure 6.

Table 3: Effect of hD on Dimensionless Pressure and Dimensionless Pressure Derivative

\begin{tabular}{|c|c|c|c|c|c|c|}
\hline $\mathrm{Td}$ & $\mathrm{PD}(\mathrm{YeD}=2)$ & $\mathrm{PD}^{\prime}(\mathrm{YeD}=2)$ & $\mathrm{PD}(\mathrm{YeD}=10)$ & $\mathrm{PD}^{\prime}(\mathrm{YeD}=10)$ & $\mathrm{PD}(\mathrm{XeD}=20)$ & $\mathrm{PD}^{\prime}(\mathrm{XeD}=20)$ \\
\hline 0.001 & 29.74 & $2.23 \mathrm{E}-01$ & 29.74 & $2.23 \mathrm{E}-01$ & 29.74 & $2.23 \mathrm{E}-01$ \\
\hline $1.00 \mathrm{E}-02$ & $2.85 \mathrm{E}+01$ & $2.10 \mathrm{E}-01$ & $2.85 \mathrm{E}+01$ & $2.10 \mathrm{E}-01$ & $2.85 \mathrm{E}+01$ & $2.10 \mathrm{E}-01$ \\
\hline 0.1 & $2.09 \mathrm{E}+01$ & $1.41 \mathrm{E}-01$ & $2.09 \mathrm{E}+01$ & $1.41 \mathrm{E}-01$ & $2.09 \mathrm{E}+01$ & $1.41 \mathrm{E}-01$ \\
\hline 1 & $1.45 \mathrm{E}+01$ & $1.05 \mathrm{E}-01$ & $1.45 \mathrm{E}+01$ & $1.05 \mathrm{E}-01$ & $1.45 \mathrm{E}+01$ & $1.05 \mathrm{E}-01$ \\
\hline 10 & $1.02 \mathrm{E}+01$ & $6.74 \mathrm{E}-02$ & $1.02 \mathrm{E}+01$ & $6.74 \mathrm{E}-02$ & $1.02 \mathrm{E}+01$ & $6.74 \mathrm{E}-02$ \\
\hline 100 & $5.92 \mathrm{E}+00$ & $4.25 \mathrm{E}-02$ & $5.92 \mathrm{E}+00$ & $4.25 \mathrm{E}-02$ & $5.92 \mathrm{E}+00$ & $4.25 \mathrm{E}-02$ \\
\hline 1000 & $5.66 \mathrm{E}+00$ & $4.25 \mathrm{E}-02$ & $5.66 \mathrm{E}+00$ & $4.25 \mathrm{E}-02$ & $5.66 \mathrm{E}+00$ & $4.25 \mathrm{E}-02$ \\
\hline 10000 & $5.66 \mathrm{E}+00$ & $4.25 \mathrm{E}-02$ & $5.66 \mathrm{E}+00$ & $4.25 \mathrm{E}-02$ & $5.66 \mathrm{E}+00$ & $4.25 \mathrm{E}-02$ \\
\hline
\end{tabular}




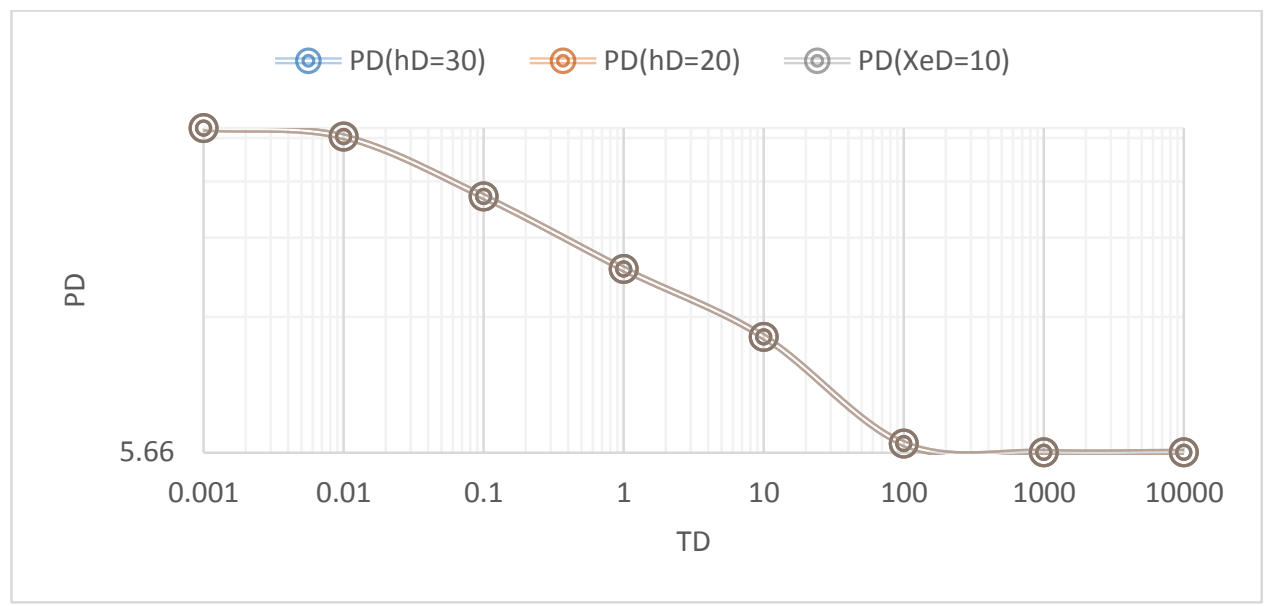

Fig. 5 Effect of $h_{D}$ on Dimensionless Pressure

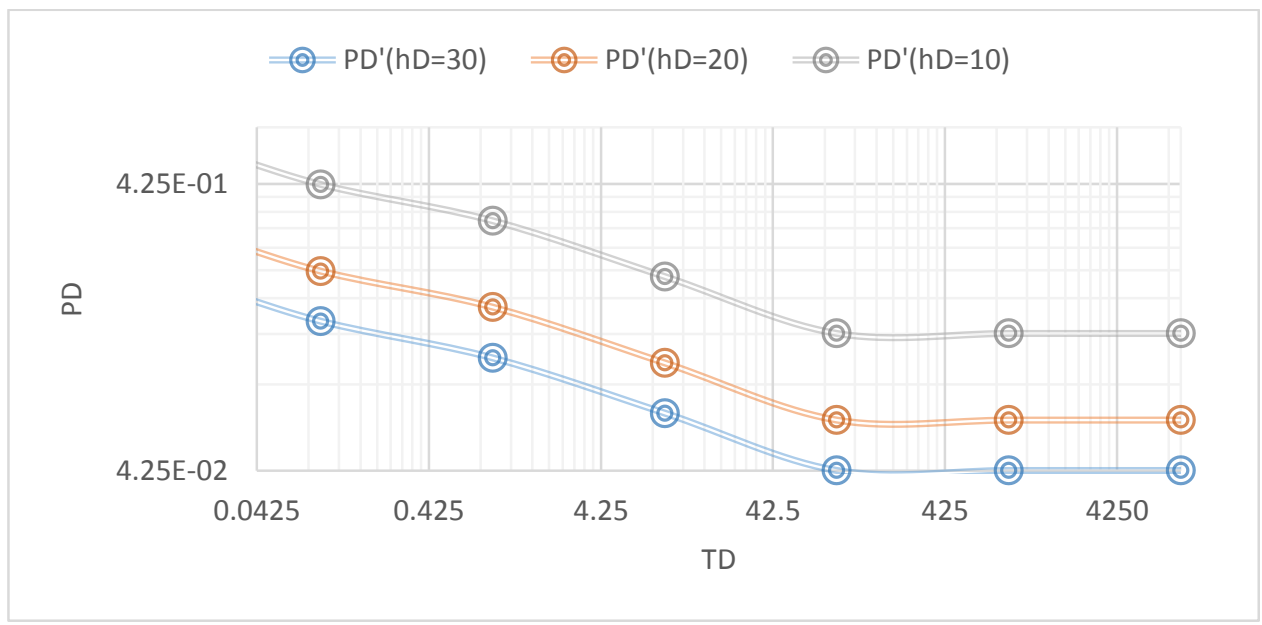

Fig. 6 Effect of $h_{D}$ on Dimensionless Pressure Derivative

\section{CONCLUSIONS}

The pressure became stable when $\mathrm{tD} \geq 100$. This behaviour is an indication that reservoir fluid has encountered a boundary.

2. It was observed that the decline rate increases with time to a point when $t D \geq 100$. At this point, the Gas cap which acts as a drive is not active. Hence, there will be no increase in production at this stage. Therefore, well can be abandon or subject to Secondary recovery.

\section{Nomenclature}

$\mathrm{P}^{\prime} \mathrm{D}=$ Pressure derivative

$\mathrm{PD}=$ dimensionless Pressure 
$\mathrm{hD}=$ dimensionless height

$\mathrm{xD}=$ arbitrary dimensionless distance along the $\mathrm{x}$-axis

$\mathrm{yD}=$ arbitrary dimensionless distance along the y-axis

$\mathrm{ZD}=$ arbitrary dimensionless distance along the z-axis

$\mathrm{zWD}=$ Well coordinate in $\mathrm{z}$-direction

$\mathrm{ywD}=$ Well coordinate in $\mathrm{y}$-direction

$\mathrm{xwD}=$ Well coordinate in $\mathrm{x}$-direction

yeD $=$ External dimensionless distance along $y$-axis

$\mathrm{xeD}=$ External dimensionless distance along $\mathrm{x}$-axis

$\mathrm{zeD}=$ External dimensionless distance along z-axis

$\mathrm{tD}=$ Dimensionless time

\section{REFERENCES}

[1] Jianzhong,O., Shiqing,C., Youwei,H., Dingyi,L., Le,L., Xinzhe.S., and Haiyang,Y., 2018, 'Diagnosis of Water-Influx Locations of Horizontal Well Subject to Bottom-Water Drive through Well- Testing Analysis", Hindawi Geofluids Volume 2018, Article ID 6385252, 1-14. https://doi.org/10.1155/2018/6385252.

[2] Oloro,J.O., and Adewole,E.S.,2019, 'Derivation of Pressure Distribution Models for Horizontal Well Using Source Function", Appl. Sci. Environ. Manage.: Vol. 23 (4), pp.575-583.

[3] Kuma,M., Sharma,P., and Gupta,D.K.,2018, 'A Sensitivity study of horizontal length, offset from water-oil contact and withdrawal rate of horizontal well in bottom water drive reservoir", J petrol Explor Prod Technol,pp.577-588

[4] Dankwa,O., Nubalanaang,S., Ahmed,G., Appau,P.O., 2018' Comparison of the Economics and Performance of Horizontal and Vertical Wells", International Journal of Petroleum and Petrochemical Engineering (IJPPE), Vol. 4, Issue 3, 2018.pp. 1-12, DOI: http://dx.doi.org/10.20431/2454-7980.0403001

[5] Gringarten,A.C., Ramey,H.J.,1973, "The Use of Source and Green's Functions in Solving Unsteady-Flow Problems in Reservoirs", SPEJ, pp. 255 285.

[6] John,M.T., Ferney,M.,James,M.S., and Saood,Q.,2019, 'Predicting Performance of High Deliverability Horizontal Gas Wells and Control of Water Cresting in Tertiary Sands East Africa", J Pet Environ Biotechnol, Vol. 10 (4),394.

[7] Jonogkittinarukom,K., and Tiab, D. ,1996,"Analysis of pressure and pressure derivative with and without type-curve matching-a horizontal well in closed- 
boundary system", Journal of Petroleum Science and Engineering, Elsevier, Petroleum Society of Canada.

[8] Kuchuk,F., Goode,P.A., Wilkinson,D.J., Thambynayagam,M.,1991, PressureTransient Behavior of Horizontal Wells with and Without Gas Cap or Aquifer, SPE,86-153. 